\title{
Interpreting English Legal Terms by Semantic Frames
}

\author{
GAO Lian-hong \\ China University of Political Science and Law, Beijing, China
}

\begin{abstract}
Legal English is characterized by the great number of legal terms, which cause difficulties for learners or translators to fully understand these legal concepts and to find equivalents in their native language. To comprehend these special terms accurately, we need to resort to semantic taxonomy and frame semantics which provide cognitive frameworks to illustrate the semantic complexities of legal terminology. It is high time as well as the first time to apply Löbner's theory of semantic taxonomy and Fillmore’s frame semantics to interpret English legal terms. According to Löbner, meanings of some linguistic unit can be analyzed into three levels: expression meaning, utterance meaning and communicative meaning, corresponding with three types of equivalency: formal, semantic and functional. Good dictionaries, as well as encyclopedias, have been trying to expose these three levels of meaning in the entries. As far as legal dictionaries are concerned, the job should be more complicated since legal terms embody unusual expression meaning though the expressions may take on common looks and the utterance meaning in addition to the functional meaning involve more intriguing legal procedures or principles. The abundance of legal language contributes to this complexity in interpreting legal terminology. Fillmore's theory and methodology of frame semantics pave the road to solving this complexity. This paper provides examples of how to use the special methodology to do the interpretation for the purpose of promoting legal literacy.
\end{abstract}

Keywords: English legal terminology, frame semantics, semantic taxonomy

\section{Introduction}

Studies concerning legal terminology have been focused upon the difficulties in translating the special terms from one language and legal culture to another. Strategies to cope with these inconveniences caused by legal terminology were usually the target of such studies. However, translation of high quality is based on good understanding or comprehension of the source text or the legal concepts, as this article is concerned. The semantic complexity of these special terms has been approached from lexical and translation perspectives, but such analysis was not to the point of the epistemological solution since no cognitive activities have been involved in the analysis. Therefore, such interdisciplinary points of view oriented in semantics and cognition are suggested to be taken in order to disentangle the complexity. These analytical underpinnings and instruments are the theories and methodological principles that are attempted to be used to analyze some legal terms and see how well the existing good knowledge, declarative or procedure, may do to promote human literacy about law, with legal concepts to start.

GAO Lian-hong, Ph.D., Asociate Professor, Department of English Language and Literature, China University of Political Science and Law, Beijing, China. 


\section{Synthesizing Studies Concerning Legal Terminology}

The research focused on translating legal terminology has been done to discuss the strategies applied when dealing with translating legal terms. The strategies were usually concerned about how to find the reliable information resources and locate the best equivalent to the term used in legal context, but the problem is, especially for less-experienced legal translators, it is difficult to tell if the equivalent is the best, or in other words, it may be best to learn how to have an accurate understanding of the term before making choices or decisions when translating. This paper is not to summarize all the strategies but point to the way to understand legal terminology. The purpose of doing such research is two-folded: on the one hand, better understanding may mean better translation, and on the other hand, learning about legal terms is the major obstacle for promoting legal literacy. Since some of the concepts may be well-known to legal practitioners but may be unfamiliar to legal translators and other laymen, it is necessary to illustrate the cognitive development of how people came to create or beaware of the significance of legal terminology.

\section{Research Development of Legal Terminology: Concepts, Theories, Terms and Terminology}

Concepts were the products of human reasoning, which symbolized great progress for human understanding of the world and academic disciplines. When concepts were increasingly testified to be comprehensive and applicable to interpret the world, subjective or objective, they might be recognized as hypotheses or even theories. Many theories were named as concepts. Without concepts, it is impossible to conduct scientific or reliable reasoning, but before reasoning, concepts need to be understood fully and accurately. This was the point made in Hohfeld'swork (Hohfeld, 1913), stressing that in legal reasoning or analysis, legal concepts were fundamental. The author himself was also a theorist acknowledged by some very practical legal practitioners, as it was widely accepted that good theories should be very useful in practice. The practice could only be well-informed by good theories or well-established concepts. In this work and later related works, concepts were usually explained by means of comparison and contrast, which mode of reasoning has been proved to be effective even till present time. Hohfeld compared and contrasted four fundamental concepts: rights, privileges, powers and immunities, and four other correlative terms: duty, no-right, liability and disability. Those two group of words were related since the first group signified legal benefits while the second group signified legal burdens. At that moment, legal terms were distinguished and explained by means of synonyms or antonyms, or "jural correlatives"and "jural opposites". The author also used definitions to explain legal terms. For example, "ownership" is defined as "the relationship between a person and any right that is vested in him” (p. 28). Then, the author further explained the relationship. He also put forward the necessity to distinguish the eight legal terms since they were often required to be clarified when the courts are often "confused by the lack of clear concepts and precise terminology".

López (1999) introduced some good dictionaries to explain legal terms, but those dictionaries were also compiled perfectly since they could not meet all the needs of legal translators, and the necessity to explain legal terms in context was emphasized by the article. Nolfi (2009) proposed that the existing legal dictionaries were not good enough for paralegals. Modern law dictionary projects were put forward when he was an associate editor at the Lawyers Co-operative Publishing Company in the mid-1980s and when he was a lead case law editor with LexisNexis in the early 2000s. His project exhibited his "interest in writing a comprehensive text that explained legal terminology in a reasonably accurate, brief, clear, and explanatory style” (p. vi), which should be valuable 
for all students of the law, especially paralegals. Mishchenko (2010), for the first time, mentioned the encyclopedic semantics in explaining legal terms, concerning the fact that concepts belonging to different legal systems are hardly ever identical and equivalents were hardly found. When teaching legal terms,

To understand what a legal concept means we have to refer to other cognitive domains which are presupposed by and incorporated in such a concept. For example, to understand the meaning of a Rights Issue we have to evoke the domain of company, shareholders, authorized capital, preemption rights etc. Besides, concepts are interrelated and embedded in various structured cultural models, cognitive models and frames which are to a certain extent reflected in national legislation and case law. (p. 5)

Her points answered López’ (1999) inquiry about explaining legal terms by contextualizing them. Cultural models, cognitive models and frames were recognized to be the contextual elements used to explain legal terms by an encyclopedic approach.

\section{From Legal Competence to Legal Literacy: The Inversion Awareness}

Legal competence means the ability to use legal rules and regulations to deal with legal conflicts, which has to be acquired by paralegals since it was required by their professionalism. Even in today's legal discourse, much emphasis is made upon how paralegals can be trained with adequate qualifications while not enough attention was given to legal literacy for other laymen though Plain Language Movement was initiated by the awareness of the importance for laymen to learn about legal rules and cases. And yet, legal terms were still too complicated and had been the major barrier for promoting legal literacy. Legal literacy seems to be less difficult to be implemented than general literacy since the former one has already got the latter one as the foundation, which means literate people may find it easier to read and understand legal rules or concepts if they were explained in plain language intelligible to them.

Legal competence requires that literate people should not only be able to understand legal concepts but also be able to study the terms and then make use of them to protect themselves from being hurt in the legal sense or just protect their own rights. In order to acquire legal competence, people need to have legal literacy as the prerequisite. As the case in other disciplines, concepts are the core knowledge to be mastered in order to conduct reasoning or analysis, which has been illustrated in previous sections. Definitions, synonyms and opposites were the means to explain legal concepts in legal dictionaries of all kind, including a legal encyclopedia. Due to the complexity of legal systems in different cultures, these tools were not adequate to teach legal English and do legal translation as well. This is the aim of this article to introduce a semantic approach to illustrate the complexity.

\section{Theoretical Underpinnings: Critical Discourse Analysis and Semantic Taxonomy}

Discourse analysis has been hovering on the ideological level no matter how complicated the analysis has been made, meaning the research studies were usually done with the linguistic reasoning missing in the argument. The issue is mainly due to the categorization and separation of disciplines, which are trying to be made independent from one another: inquiries about linguistic forms and semantic analysis get extended into the philosophical field while discourse analysis was mainly conducted in cultural and political studies to be focused on ideological pursuits. However, semantics, as it is placed in the middle to connect linguistic forms with ideological meanings, is the platform to exhibit theories showing the relationship among forms, meanings and ideas. To do discourse reasoning means to start from forms and seek the associations between the three levels. 
Critical discourse analysis has to be based upon such logical and reliable reasoning since it is conducted for the purpose of social transformation. That's why the term "critical" is used, meaning to be responsible for fact findings and subsequently social change for the better.

Semantics is the field where meanings of a linguistic unit are analyzed. According to Löbner (2013), meanings of some linguistic unit can be analyzed into three levels: expression meaning, utterance meaning and communicative meaning, corresponding with three types of equivalency: formal, semantic and functional. Expression meaning refers to the meaning of morphemes, derivatives, compounds, phrases, clauses and sentences, so expression meaning analysis combined morphological, lexical and syntactic analysis with the semantic research, which is purely concerned with the meanings of these linguistic forms. Utterance meaning refers to the indices that may influence the meanings of the forms, such as the author of the context, the reader, time, place and facts. Two concepts are also used to do the analysis: reference and truth. The truthfulness of the sentences is often studied by studying the referential relations (Löbner, 2013). Utterance meaning also includes the meaning predictions and implicatures made by the author and reader based upon the context. This part of the semantic inquiry extends itself into the field of pragmatics and gets connected with the last type of meaning: communicative meaning. Communicative meaning refers to the interaction between context and linguistic forms, or how the semantic judgments made by the conversation participants impact the communication progress and the participants' behaviors. Pragmatic scholars study the semantic negotiations between text producers and recipients, the utterance context and implicatures (Thomas, 2014). The semantic negotiation is based on the communication participants' understanding of the semantic and logical associations between the linguistic forms and grammatical relations in addition to the cultural or social rules.

\section{The Methodological Approach for Semantic Analysis of Legal Terminology: Semantic Frames}

Semantic analysis by means of semantic frames is to approach the meanings of a linguistic unit from different dimensions so as to expose the connotations and denotations of some lexical units or words which stand for concepts or even theories. Those dimensions include such concrete situational elements as characters, behaviors, approaches, conditions, states and causes, constituting the cognitive frames for people to fully understand the concepts (Fillmore, 2006). It may not necessary to learn general words used in everyday life, but it should be required to learn legal concepts by this approach due to the complexity of legal systems in cross-cultural legal communications.

\section{Definition, Opposites, Synonyms, Proposition, Concepts and Framework}

To explain the meanings of a word has undergone a long process of development. Originally, linguistic scholars tried to explain the meanings with definitions, i.e. using much simpler words to explain more complicated words, sometimes complemented by providing opposites and synonyms, in order to remind people of similar or familiar words to scaffold the learning of more complicated lexical units. Epistemological research gave rise to more academic concepts, which helped people to learn about the world in more systematic and reliable ways. "proposition" is the concept stressing that utterance should contain some contents, which can be consistent with the reality or not, or true or false. Some true propositions may turn out to be the contents of some concepts, which has been recognized to be truthful in exposing some reliable principles to explore the world. If 
the contents were many-folded, theories or theoretical frameworks were created. This gradual progress in human's cognition demonstrates different ways to explore the meanings of words, from simple to very complicated, dependent on the contexts in which words or concepts are used.

\section{Building the Framework}

Here are some good examples used by linguistic scholars to illustrate the application of semantic frames to explain lexical meanings.

Game

Opponents

Played for fun

Winners and losers

Luck and skills needed

The four dimensions of "game" summarize most of the possible contextual elements for people to understand the meanings of the word. The cognition is based on establishing relations between language, life and human experiences. Human experiences associated life with experiences; people learn about life by means of their own experiences. Semantic frames are helpful for human cognition since the approach of linguistic analysis is in the consistency of such a cognitive principle: people learn by experiencing.

Commercial Transaction

Seller

Buyer

Goods

Exchange of money

The frame is composed of the possible elements involved in the commercial activity. Smuggle

Perpetrator

Goods

Goal

Source

Path

From the frame, people can learn about all the factors that constitute the crime. These aspects are the perspectives where law enforcers can search for evidence based on which to make tentative judgments on whether it can be recognized as illegal activities or not.

\section{Research Findings: A Comparative Corpus-based Example}

Legal textbooks for the purpose of promoting legal literacy were usually written in conceptual frameworks, with most of the chapter titles as concepts, all of which constitute the whole legal system. In this article, two of the books are cited and the concepts explained in them are compared as they are analyzed in the books (Martin, 2011; Nolfi, 2009). The two books exhibit the English legal system in their own ways: 
Table 1

Comparison of the Interpretation of the English Legal System by the Two Textbooks

\begin{tabular}{|l|l|}
\hline Martin 2011 & Nolfi 2009 \\
\hline Chapter 1: What is law? & Part 1: Law \\
\hline Chapter 2: Judicial precedent & Part 2: People in law \\
\hline Chapter 3: Legislation & Part 3: Constitutional law and government \\
\hline Chapter 4: European Union law & Part 4: Civil law: status issues \\
\hline Chapter 5: Law reform & Part 5: Civil law: business issues \\
\hline Chapter 6: The civil justice system & Part 6: Civil law: social issues \\
\hline Chapter 7: Tribunals and inquiries & Part 7: Civil law: procedure \\
\hline Chapter 8: Police powers & Part 8: Criminal law: substantive issues \\
\hline Chapter 9: The criminal process and courts & Part 9: Criminal law: procedure \\
\hline Chapter 10: Sentencing & Part 10: Legal research and writing \\
\hline Chapter 11: The legal profession & \\
\hline Chapter 12: The Judiciary & \\
\hline Chapter 13: Lay people in the legal system & \\
\hline Chapter 14: Legal services and funding & \\
\hline
\end{tabular}

Under Nolfi's (2009) 10 parts, there are 57 chapters to introduce the legal system in a more substantial way. The two books' chapters are actually the conceptual frameworks to present what the legal system is like in the authors' minds. By this example, we may find that semantic frame may be very substantial, dependent on what the term is. Constructing such frames may need to resort to information resources most of the times regarded as some form of the corpus, which should be the best way to find all the possible related elements to build the frames.

\section{Reflection Upon the Study}

The most fundamental way to build up legal literacy or competence is to write and compile good legal dictionaries and encyclopedia. Facilitated by electronic technology and universal awareness of the usefulness and meaningfulness of these legal resources, more and more people should be able to be attracted to this great work, which can be developed into the largest knowledge pool or sea for legal learners and researchers. To do this, global attention and some universal language system, verbal or technical, are to be needed in constructing theholistic culture for legal purposes, which should be part of human culture, and in turn, encompasses everything from culture. This advocate is initiated by understanding "good law" not just as rules to punish wrong behaviors or prevent people from behavioral deviations but as cultural conventions for people to revere for and abide by, just as people observe festival conventions. At that time, legal competence should have been converted into cultural competence, when it should be not difficult to acquire legal competence, as easy as enculturation into a native community.

\section{References}

Fillmore, C. (2006). Frame semantics. In D. Geeraerts (Ed.), Cognitive linguistics: Basic readings (pp. 373-400). Berlin: Mouton de Gruyter.

Hohfeld, W. N. (1913). Some fundamental legal conceptions as applied in judicial reasoning. The Yale Law Journal, $23(1), 16-59$. Löbner, S. (2013). Understanding semantics. New York, London: Routledge.

López, A. S. (1999). Translating legal terms in context. Legal Reference Services Quarterly, 17.4, 105-113. 
Mishchenko, V. (2010). Terminology translation in teaching legal English. English for Specific Purposes World, 9(29), 6. Martin, J. (2011). Key facts: The English legal system (4th ed.) UK: Hodder Education.

Nolfi, A. E. (2009). Legal terminology explained. New York, NY: McGraw-Hill.

Thomas, J. (2014). Meaning in interaction: An introduction to pragmatics. New York, London: Routledge. 